\title{
BLOW-UP TIME ESTIMATES FOR A NON-LOCAL REACTIVE-CONVECTIVE PROBLEM MODELLING STERILIZATION OF FOOD
}

\author{
C. V. NIKOLOPOULOS \\ Department of Mathematics, University of the Aegean, Samos, Greece \\ E-mail: cnikolo@aegean.gr \\ D. E. TZANETIS \\ Department of Mathematics, Faculty of Applied Sciences \\ National Technical University of Athens, 15780 Athens, Greece \\ E-mail:dtzan@math.ntua.gr
}

1. Introduction. We consider the non-local initial boundary value problem

$$
\begin{gathered}
u_{t}(x, t)+u_{x}(x, t)=\lambda \frac{f(u(x, t))}{\left(\int_{0}^{1} f(u(x, t)) d x\right)^{2}}, \quad 0<x<1, \quad t>0, \\
u(0, t)=0, t>0, \\
u(x, 0)=u_{0}(x) \geq 0, \quad 0<x<1,
\end{gathered}
$$

where $\lambda>0$. The function $u(x, t)$ represents the dimensionless temperature when an electric current flows through a conductor (e.g. food) with temperature dependent on electrical resistivity $f(u)>0$, subject to a fixed potential difference $V>0$. The (dimensionless) resistivity $f(u)$ may be either an increasing or a decreasing function of temperature depending strongly on the type of the material (food). Problem (1) models one of the main methods for sterilizing food. The sterilization can take place by electrically heating the food rapidly.

Here $\lambda$ is a dimensionless parameter and can be identified, amongst other things, with the square (being actually proportional) of the applied potential difference $V$. In the case where $f(u)$ is a sufficiently rapidly decreasing function of temperature, there exists a critical value of the potential difference $V$, say $V^{*}$, such that for $V>V^{*}$ (equivalently

2000 Mathematics Subject Classification: Primary 35L60; Secondary 35Q80.

Research of the first author supported by Thalis grant 65/132400, NTUA, Greece.

The paper is in final form and no version of it will be published elsewhere. 
(a)

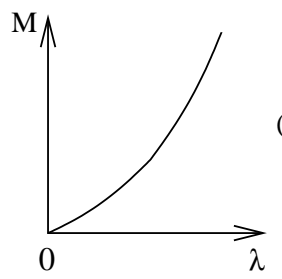

(b)

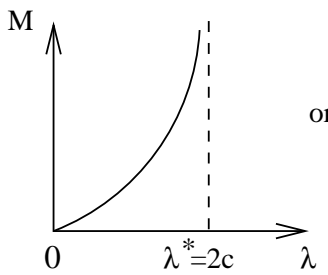

or

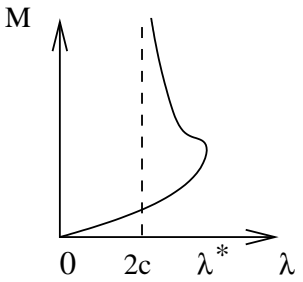

(c)

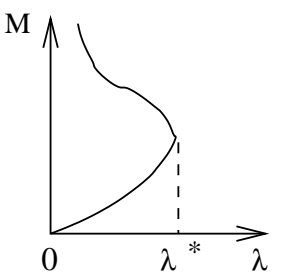

Fig. 1. Possible response diagrams for equilibrium solutions: (a) $M f(M)$ unbounded; (b) $M f(M) \rightarrow c, 0<c<\infty$,as $M \rightarrow \infty ;($ c) $M f(M) \rightarrow 0$, as $M \rightarrow \infty$. Each diagram may contain more turning points than shown (so that for some $\lambda$ there are more solutions).

$\lambda>\lambda^{*}$ ) a thermal runaway (blow-up of the temperature $u$ or burning of the food) takes place, see $[6,7,8]$.

In the following we assume $f$ to satisfy

$$
\begin{gathered}
f(s)>0, \quad f^{\prime}(s)<0, \quad s \geq 0 \quad \text { and } \\
\int_{0}^{\infty} f(s) d s<\infty,
\end{gathered}
$$

for instance either $f(s)=e^{-s}$ or $f(s)=(1+s)^{-p}, p>1$, satisfy (2).

For the initial data it is required that $u_{0}(x), u_{0}^{\prime}(x)$ be bounded, $u_{0}(x) \geq 0$ in $[0,1]$ (this is a consequence of the fact that for any initial data the solution $u$ becomes nonnegative over $(0,1]$ for some time $t$ and so, with an appropriate redefinition of $t$, the last requirement can always be assumed, $[6,8])$, and in some cases $u_{0}(x) \rightarrow 0+$ properly as $x \rightarrow 0+$.

The solution $u(x, t)$ also blows up for large enough initial data even if $0<\lambda \leq \lambda^{*}$, $[6,7,8]$; in this case, an analogous estimate of blow-up time, as for $\lambda>\lambda^{*}$, will be given in a future paper.

The steady problem corresponding to (1) is

$$
w^{\prime}=\mu f(w)=\lambda \frac{f(w)}{\left(\int_{0}^{1} f(w) d x\right)^{2}}, \quad 0<x<1, \quad w(0)=0,
$$

where $w=w(x)=w(x ; \lambda)$ (see $[1,2,6,7,8]$ ). The parameter $\mu$ is referred to as a local parameter while $\lambda$ as a non-local one and the relation between them is $\mu=$ $\lambda /\left(\int_{0}^{1} f(w) d x\right)^{2}$.

In the present work, our purpose is to find some estimates of the blow-up time $t^{*}$, with respect to the parameter $\lambda$ (more precisely, with respect to the difference $\lambda-\lambda^{*}$ ) when $\lambda>\lambda^{*}$, and fixed initial data $u_{0}(x)$. 
In Figure 1, (c) or (b) with $2 c<\lambda^{*}$, there may be either only one or more than one turning point $\left(\lambda^{*}, M^{*}\right)$ depending on $f$. One can find other forms of non-local diagrams in $[6,7,8]$; their shapes depend strongly on boundary conditions and the function $f$.

Under the assumptions (2), problem (3) has at least one classical (regular) steady solution $w^{*}=w\left(x ; \lambda^{*}\right)$, (more than one $w^{*}$ may exist). In the following, we assume that $w^{*}$ is unique, i.e. Figure $1(\mathrm{c})$, and that the pair $(\underline{w}, \bar{w})$ at $\lambda<\lambda^{*}\left(\lambda\right.$ close to $\left.\lambda^{*}\right)$ has the property: $\underline{w}=w_{1}$ is stable while $\bar{w}=w_{2}$ is unstable, (since in our proofs we require only the existence of at least one $w^{*}$ at $\lambda^{*}$ and that $\underline{w}(x)<\bar{w}(x)$ for $x$ in $(0,1]$ where $\bar{w}$ is the next steady solution greater than $\underline{w}(x))$ at $\left.\lambda<\lambda^{*}\right)$.

We also emphasize that for $\lambda>\lambda^{*}$ and for all $x \in(0,1]$ we have:

$$
\begin{gathered}
F(u)=\frac{f(u)}{\left(\int_{0}^{1} f(u) d x\right)^{2}} \rightarrow \infty \text { as } t \rightarrow t^{*}-<\infty, \\
u(x, t ; \lambda) \rightarrow \infty \text { as } t \rightarrow t^{*}-<\infty,
\end{gathered}
$$

the latter means that $u(x, t ; \lambda)$ blows up globally, see $[6,7,8]$.

We organize this work as follows: in Section 2 we apply comparison techniques and find upper and lower bounds for $t^{*}$, when $f$ satisfies (2). In Section 3, we use an asymptotic expansion and again obtain an estimate of $t^{*}$ but for $f(s)=e^{-s}$. Also we numerically compute the blow-up time $t^{*}$ using an up-wind scheme and verifying the previous estimate.

2. Comparison methods: upper and lower bounds of $t^{*}$ for $\lambda>\lambda^{*}$. If the function $f$ satisfies (2a), one can prove (see appendix in [8]) that a maximum principle holds for (1) (here is where we need $f$ to be decreasing). Then we may, in the usual way, define upper and lower solutions of (1): an upper (lower) solution $\bar{u}(\underline{u})$ is defined as a function which satisfies $(1)$ if we substitute $\geq(\leq)$ for $=$, see $[6,7,8,9,10]$. In the following work, we use ideas and techniques similar to [3].

2.1. An upper bound for $t^{*}$. We now wish to find an upper bound for the blow-up time $t^{*}$. For simplicity, we assume that $0 \leq u_{0}<w^{*}$. Firstly, we write $(3)$ in a slightly different way,

$$
w^{\prime}=\mu f(w)=\frac{\lambda f(w)}{\left(\int_{0}^{1} f(w) d x\right)^{2}}=\lambda F(w), \quad 0<x<1, \quad w(0)=0,
$$

where $F(\cdot)=f(\cdot) /\left(\int_{0}^{1} f(\cdot) d x\right)^{2}$ and $\lambda$ is a positive parameter (eigenvalue). Then, the related linearized eigenvalue problem of (5) for a function $\phi=\phi(x ; \lambda) \in \mathbb{R}$ (actually $\phi$ is assumed to be a real function) is:

$$
\phi^{\prime}-\lambda \delta F(w ; \phi)=-\rho(w, \lambda) \phi, \quad 0<x<1, \quad \phi(0)=\phi_{0}=0,
$$

where $\delta F(w ; \phi)$ is the first variation (or Gâteaux derivative) of $F$ at $w$ in the direction of $\phi,\left(F(w ; \phi):=F(w+\epsilon \phi)=J(\epsilon)\right.$ and $\left.\delta F(w ; \phi)=J^{\prime}(0)=\lim _{\epsilon \rightarrow 0} \frac{F(w+\epsilon \phi)-F(w)}{\epsilon}\right)$.

As regards the first variation $\delta F(w ; \phi)$ we have

$$
\delta F(w ; \phi)=\frac{f^{\prime}(w) \phi}{\left(\int_{0}^{1} f(w) d x\right)^{2}}-\frac{2 f(w) \int_{0}^{1} f^{\prime}(w) \phi d x}{\left(\int_{0}^{1} f(w) d x\right)^{3}} .
$$


In the following, in order to simplify the expressions, we use the notation:

$$
I_{\nu k}(w, \phi):=\int_{0}^{1} f^{(\nu)}(w(x)) \phi^{k}(x) d x,
$$

and $I_{\nu}(w):=I_{\nu 0}(w, \phi), \nu, k=0,1,2,3, \ldots, f^{(\nu)}(w)=\frac{d^{\nu}}{d w^{\nu}} f(w)$, thus

$$
\delta F(w ; \phi)=\frac{f^{\prime}(w) \phi}{I_{0}^{2}(w)}-\frac{2 f(w) I_{11}(w, \phi)}{I_{0}^{3}(w)} .
$$

Now we can have the following lemma concerning the eigenpair of problem (6).

Lemma 1. Problem (6) has the eigenpair $(\rho, \phi)$ where $\phi(x)>0$ in $(0,1]$ and its spectrum is a continuum of eigenvalues in $\mathbb{R}$, generated by $\rho=\rho(w, \lambda)$ for every $\lambda \in\left(0, \lambda^{*}\right]$. The function $\rho(w, \lambda)$ is continuous with respect to $\lambda$.

Proof. If $\phi(x)$ is a real function then equation (6) implies that $\rho \in \mathbb{R}$. Now equation (6) can be written in a different way

$$
\phi^{\prime}+(g(x)+\rho) \phi=q h(x)
$$

where $q=-I_{11}(w, \phi)$ is a number, $h(x)=\lambda \frac{2 f(w)}{I_{0}^{3}(w)}>0$ and $g(x)=-\lambda \frac{f^{\prime}(w)}{I_{0}^{2}(w)}$. Also, problem (6) has the following integral representation:

$$
\phi(x)=q\left[\exp \left(-\int_{0}^{x} g(z) d z-\rho x\right)\right] \int_{0}^{x} h(s)\left[\exp \left(\int_{0}^{s} g(z) d z+\rho s\right)\right] d s .
$$

The above form of $\phi$ implies that if a nontrivial $\phi$ satisfying (6) exists, then it is positive ( $\phi$ actually does not change sign in $(0,1)$ and can be taken as positive).

Now we can normalize $\phi$ so that $q=1$. Therefore, there exists a function $\phi \in \mathbb{R}$ satisfying (6) and the following equation:

$$
1=-I_{11}(w, \phi)=\int_{0}^{1}-f^{\prime}(w) \phi(x) d x, \quad \text { where } \quad \phi(x)=\phi(x ; \rho) .
$$

This can be written as

$$
1=\int_{0}^{1}-f^{\prime}(w)\left[\int_{0}^{x} h(x-s) \exp [-\rho s-G(x)+G(x-s)] d s\right] d x
$$

for $G(s)=\int_{0}^{s} g(z) d z$. Therefore

$$
1=\int_{0}^{1} \exp (-\rho s)\left[\int_{s}^{1}\left(-f^{\prime}(w) h(x-s) \exp [-G(x)+G(x-s)]\right) d x\right] d s \quad \text { or }
$$

$$
1=\int_{0}^{1} Y(s) \exp (-\rho s) d s
$$

where $Y(s)=Y(s ; w)>0$ for $-f^{\prime}(w) h(x-s) \exp [-G(x)+G(x-s)]>0$ and $0<s<$ $x<1$, (the eigenvalue $\rho$ is a real number). Now for $Y(s)>1$ we have only one real solution $\rho>0$ which satisfies equation (7). Also for $Y(s)<1$ we have only one real solution $\rho<0$ and for $Y(s)=1$ we have only the trivial solution $\rho=0$. The eigenvalues $\rho=\rho(w, \lambda) \in C\left(\left(0, \lambda^{*}\right] ; \mathbb{R}\right)$ since the function $w(x, \lambda), g(x)=g(x ; \lambda)$ and $Y(s)=Y(s ; \lambda)$ are continuous functions with respect to $\lambda \in\left(0, \lambda^{*}\right]$. This proves the lemma. 
It is known that the spectrum to problem (5) can be either an interval closed from the right, or an open one. Here, we consider the case where the spectrum to problem (5) is an interval closed from the right and that there exists a unique turning point $\left(\lambda^{*},\left\|w^{*}\right\|_{\infty}\right)$ with $\left\|w^{*}\right\|_{\infty}=w^{*}(1)=M^{*}<\infty$, see Figure $1(\mathrm{c})$; at $\lambda<\lambda^{*}$ two steady states correspond $w_{1}, w_{2}$ with $w_{1}<w_{2}$, while at $\lambda=\lambda^{*}$ correspond $w_{1}=w_{2}=w^{*}$. We need the following:

LEMma 2. Let $w_{1}, w_{2}$ with $w_{1}<w_{2}$ be the solutions of (5) at $\lambda<\lambda^{*}$, then $\rho_{1}=$ $\rho\left(w_{1}, \lambda\right) \leq 0, \rho_{2}=\rho\left(w_{2}, \lambda\right) \geq 0$ and $\rho^{*}=\rho\left(w^{*}, \lambda^{*}\right)=0$ where $\rho$ represents the eigenvalues of problem (6).

Proof. We assume $u(x, t)=w(x)+\epsilon \phi(x) e^{\rho t}+O\left(\epsilon^{2}\right)$, with $\phi(x)>0$, for $x \in(0,1]$ and for some $\epsilon \in \mathbb{R}$. Then we find, to the first order of $\epsilon$, that $\phi$ and $\rho$ must satisfy problem (6). We also know that $w_{1}$ is asymptotically stable, $w_{2}$ is unstable and $w^{*}$ is stable from below and unstable from above, see [8]. This implies at least that $\rho_{1} \leq 0, \rho_{2} \geq 0$ and $\rho^{*}=0$.

Because of Lemmas 1 and 2, problem (6) at $\lambda=\lambda^{*}$, with $\phi^{*}(x)>0$, becomes

$$
\phi^{* \prime}-\lambda^{*} \delta F\left(w^{*} ; \phi^{*}\right)=0, \quad 0<x<1, \quad \phi^{*}(0)=\phi_{0}^{*}=0 .
$$

Now, in order to find an upper bound for $t^{*}$, we take the difference

$$
v=v(x, t)=v(x, t ; \lambda)=u(x, t ; \lambda)-w^{*}(x)=u-w^{*} .
$$

Since $w^{*}$ is bounded, $v$ blows up at the same time as $u$ does and in the same manner, i.e. globally. Hence $t^{*}=t^{*}(u)=t^{*}(v)\left(t^{*}(u)\right.$ is the blow-up time for $\left.u\right)$ and $v(x, t) \rightarrow \infty$ as $t \rightarrow t^{*}-$ for all $x \in(0,1]$. In the following, we find an $A$-problem (see below (15)), where $A=A(t)$ blows up and is such that: $A(t) \leq c\|v(\cdot, t)\|_{\infty}$ where $c=1 / \sup _{x} \phi^{*}(x)$ as long as $v(x, t) \geq \psi(x, t)=A(t) \phi^{*}(x)$. The latter relation and (9) imply $t^{*}(u)=t^{*}(v) \leq T^{*}=$ $T^{*}(A)$, for some $T^{*}$, thus we find an upper bound $T^{*}$ for $t^{*}(u)$.

Therefore, we obtain

$$
v_{t}=u_{t}=-v_{x}+\left(\lambda-\lambda^{*}\right) F(u)+\lambda^{*}\left(F(u)-F\left(w^{*}\right)\right) .
$$

By writing $J(\epsilon)=F\left(w^{*}+\epsilon v\right), 0 \leq \epsilon \leq 1$, whence $J(0)=F\left(w^{*}\right)$ and $J(1)=F(u)$, Taylor's formula gives $F(u)-F\left(w^{*}\right)=J(1)-J(0)=J^{\prime}(0)+\frac{J^{\prime \prime}(\xi)}{2}$, for some $\xi=\xi(t) \in$ $(0,1)$, where $J^{\prime}(0)=\delta F\left(w^{*} ; v\right)=\left[\frac{d}{d \epsilon} J(\epsilon)\right]_{\epsilon=0}$. Also

$$
\delta^{2} F(z ; v)=\frac{f^{\prime \prime}(z) v^{2}}{I_{0}^{2}(z)}-\frac{4 v f^{\prime}(z) I_{11}(z, v)}{I_{0}^{3}(z)}-\frac{2 f(z) I_{22}(z, v)}{I_{0}^{3}(z)}+\frac{6 f(z) I_{11}^{2}(z, v)}{I_{0}^{4}(z)}
$$

where $z=w^{*}+\xi v$ and $\delta^{2} F(z ; v)=J^{\prime \prime}(\xi)$ is the second Gâteaux derivative. Thus, from equation (10) and setting $v=u-w^{*}=\theta \hat{v}$, for $0<\theta=\lambda-\lambda^{*} \ll 1$, we get the problem:

$$
\theta \hat{v}_{t}+\theta \hat{v}_{x}=\theta F(u)+\lambda^{*} \theta \delta F\left(w^{*} ; \hat{v}\right)+\frac{\lambda^{*}}{2} J^{\prime \prime}(\xi), 0<x<1, t>t_{1},
$$

$$
\begin{gathered}
\hat{v}(0, t)=0, \quad t>t_{1}, \\
\theta \hat{v}\left(x, t_{1}\right)=u\left(x, t_{1}\right)-w^{*}(x) \geq 0, \quad 0<x<1 .
\end{gathered}
$$


This is simplified to

$$
\hat{v}_{t}+\hat{v}_{x}=F(u)+\lambda^{*} \delta F\left(w^{*} ; \hat{v}\right)+\frac{\lambda^{*}}{2} \theta \hat{J}^{\prime \prime}(\xi), \quad 0<x<1, \quad t>t_{1},
$$

where $J(\xi)=\theta^{2} \hat{J}(\xi)=\theta^{2} \delta^{2} F(z ; \hat{v})$. Now we find a lower solution $\psi$ for $\hat{v}$-problem (11). Therefore, we require $\psi=\psi(x, t)$ to satisfy

$$
\psi_{t}+\psi_{x} \leq F(u)+\lambda^{*} \delta F\left(w^{*} ; \psi\right)+\frac{\lambda^{*}}{2} \theta \delta^{2} F(z ; \psi) .
$$

Setting $\psi(x, t)=A(t) \phi^{*}(x)$ and $\dot{A}(t)=\frac{d}{d t} A(t)$ we obtain

$$
\dot{A}(t) \phi^{*}+A(t) \phi^{* \prime}-\lambda^{*} A(t) \delta F\left(w^{*} ; \phi^{*}\right) \leq F(u)+\frac{\lambda^{*}}{2} \theta \delta^{2} F(z ; \psi) .
$$

Using $\phi^{*}$-problem (8), relation (4) (there exists $\beta$ such that $\beta A(t) \phi^{*} \leq F(u) \rightarrow \infty$ for $t$ close to $t^{*}$ ), and equation (13), it is enough to consider

$$
\dot{A}(t) \phi^{*} \leq \beta A(t) \phi^{*}+\frac{\lambda^{*}}{2} \theta \delta^{2} F(z ; \psi) \leq F(u)+\frac{\lambda^{*}}{2} \theta \delta^{2} F(z ; \psi) .
$$

Similarly, for $0<\theta \ll 1$, i.e. $\lambda$ close to $\lambda^{*}$, we can find $\beta_{1}>0$ so that we get

$$
\dot{A}(t) \phi^{*} \leq \beta_{1} A(t) \phi^{*} \leq \beta A(t) \phi^{*}+\frac{\lambda^{*}}{2} \theta A^{2}(t) \delta^{2} F\left(z ; \phi^{*}\right), \quad t>\tau_{1} .
$$

Taking $c$ small enough so that $\theta \leq \frac{c}{A(t)}$ (for some fixed $\theta$ we choose $c$ and $c_{1}$, see below, so that $\theta \leq \frac{c}{A(t)}$ where $c$ is about the time that $u$ is smaller than order one i.e. $u(x, t)$ is bounded, $A(\tau) \phi^{*} \theta+w^{*} \leq u(x, \tau)$ and $\left.0<t^{*}-\tau \ll 1\right)$, thus we have that $A(t) \leq c_{1} e^{\beta_{1} t} \leq \frac{c}{\theta}$ with $c_{1}=A\left(\tau_{1}\right) e^{-\beta_{1} \tau_{1}}$ and this holds for time $t=\tau=\frac{1}{\beta_{1}} \ln \left(\frac{c}{\theta c_{1}}\right)$. Now we can obtain an upper estimate $T_{u}^{*}$ for $t^{*}(u)$ which is $T_{u}^{*}=\tau+t_{1}^{*}>t^{*}(u)=t^{*}$, where $t_{1}^{*} \ll \tau$ is the blow-up time of the problem (1) for $t>\tau$ and $u(x, \tau)=w^{*}+c \phi^{*} \geq 0$, $0<x<1$.

2.2. A lower bound for $t^{*}$. We take $u_{0}(x)$ such that $u_{0}(x)<w^{*}(x)$ for $0<x<1$ and $u_{0}(0)=w^{*}(0)=0$. Let $u^{*}=u^{*}(x, t)=u\left(x, t ; \lambda^{*}\right)$ be the solution to $(1)$ with $u_{0}^{*}=u_{0}$.

In the following we use a similar concept to those in $[3,4]$. Therefore we set $u=$ $u^{*}+u_{1} \leq u^{*}+\psi_{1}=w^{*}-\hat{u}+\psi_{1} \leq w^{*}-\psi+\psi_{1}<w^{*}<\infty$, where $\hat{u}$ is given by $\hat{u}=w^{*}-u^{*}>0$ and satisfies (16), $u_{1}$ solves (23) (see below), $\psi_{1}$ is an upper solution to the $u_{1}$-problem and $\psi$ is a lower solution to the $\hat{u}$-problem, i.e. $\psi_{1} \geq u_{1}$ and $\psi \leq \hat{u}$. The $\hat{u}$-problem is defined by

$$
\begin{gathered}
\hat{u}_{t}=-\hat{u}_{x}-\lambda^{*}\left(F\left(u^{*}\right)-F\left(w^{*}\right)\right), 0<x<1,0<t<T, \\
\hat{u}(0, t)=w^{*}(0)-u^{*}(0, t)=0,0<t<T, \\
\hat{u}(x, 0)=\hat{u}_{0}(x)=w^{*}(x)-u_{0}^{*}(x), 0<x<1,
\end{gathered}
$$

with $\hat{u}_{0}>0$, hence $\hat{u}>0$, for $0<x<1,0<t<T<t^{*}$ and for some $T>0$. We write $J(\epsilon)=F\left(w^{*}-\epsilon \hat{u}\right), 0 \leq \epsilon \leq 1$ and examine the difference,

$$
F\left(u^{*}\right)-F\left(w^{*}\right)=J(1)-J(0)=J^{\prime}(0)+\frac{J^{\prime \prime}(\xi)}{2}, \quad 0<\xi<1,
$$


where $J^{\prime}(0)=\delta F\left(w^{*} ;-\hat{u}\right)=-\delta F\left(w^{*} ; \hat{u}\right)=-\left(\frac{f^{\prime}\left(w^{*}\right) \hat{u}}{I_{0}^{2}\left(w^{*}\right)}-\frac{2 f\left(w^{*}\right) I_{11}\left(w^{*}, \hat{u}\right)}{I_{0}^{3}\left(w^{*}\right)}\right)$ and

$$
\begin{aligned}
J^{\prime \prime}(\xi)= & \delta^{2} F(z ;-\hat{u})=\delta^{2} F(z ; \hat{u})=\frac{f^{\prime \prime}(z) \hat{u}^{2}}{I_{0}^{2}(z)}-\frac{4 \hat{u} f^{\prime}(z) I_{11}(z, \hat{u})}{I_{0}^{3}(z)} \\
& -\frac{2 f(z) I_{22}(z, \hat{u})}{I_{0}^{3}(z)}+\frac{6 f(z) I_{11}^{2}(z, \hat{u})}{I_{0}^{4}(z)}
\end{aligned}
$$

with $0<z=w^{*}-\xi \hat{u}<w^{*}<\infty$, for some $\xi=\xi(t) \in(0,1)$.

Thus, equation (16a) and (17) give:

$$
\mathrm{L}(\hat{u}):=\hat{u}_{t}+\hat{u}_{x}-\lambda^{*} \delta F\left(w^{*} ; \hat{u}\right)+\frac{\lambda^{*}}{2} \delta^{2} F(z ; \hat{u})=0 .
$$

Now we introduce the function $\psi=\psi(x, t)=\frac{c \phi^{*}(x)}{t+t_{0}}+\frac{u_{2}(x)}{\left(t+t_{0}\right)^{2}}$, where $c, t_{0}$ (positive constants), $u_{2}=u_{2}(x) \geq 0$ are to be determined and $\phi^{*}=\phi^{*}(x)$ satisfies problem (8). For $u^{*}=w^{*}-\psi-r=w^{*}-\frac{c \phi^{*}(x)}{t+t_{0}}-\frac{u_{2}(x)}{\left(t+t_{0}\right)^{2}}-r \geq 0$, where $r=r(x, t)=\frac{u_{3}(x)}{\left(t+t_{0}\right)^{3}}+\frac{u_{4}(x)}{\left(t+t_{0}\right)^{4}}+\ldots$, $(r \in \mathbb{R})$, since $u^{*} \rightarrow w^{*}-$ or $(\psi+r) \rightarrow 0+$ as $t \rightarrow \infty\left(u_{0}^{*}(x)<w^{*}(x)\right)$, actually $u^{*}<w^{*}$ and $\psi+r>0$ for all $t \geq 0$, [8]. Thus, by using Taylor's expansion for the term $F\left(u^{*}\right)$, the equation $u_{t}^{*}+u_{x}^{*}=\lambda^{*} F\left(u^{*}\right)$ becomes

$$
\begin{aligned}
& \frac{c \phi^{*}}{\left(t+t_{0}\right)^{2}}+w^{* \prime}-\frac{c \phi^{* \prime}}{t+t_{0}}-\frac{u_{2}^{\prime}}{\left(t+t_{0}\right)^{2}}+\cdots=\lambda^{*} F\left(w^{*}-\frac{c \phi^{*}}{t+t_{0}}-\frac{u_{2}}{\left(t+t_{0}\right)^{2}}+\ldots\right)= \\
& \lambda^{*} F\left(w^{*}\right)-\lambda^{*} \frac{c}{t+t_{0}} \delta F\left(w^{*} ; \phi^{*}\right)-\frac{\lambda^{*}}{\left(t+t_{0}\right)^{2}}\left[\delta F\left(w^{*} ; u_{2}\right)-\frac{c^{2}}{2} \delta^{2} F\left(w^{*} ; \phi^{*}\right)\right]+\ldots
\end{aligned}
$$

Equating terms of the same order with respect to powers of $\frac{1}{t+t_{0}}$, taking into account the $\phi^{*}$-problem, we have

$$
\left(c \phi^{*}-u_{2}^{\prime}\right)=\frac{\lambda^{*}}{2} c^{2} \delta^{2} F\left(w^{*} ; \phi^{*}\right)-\lambda^{*} \delta F\left(w^{*} ; u_{2}\right) .
$$

Then we choose $c$ so that $c \int_{0}^{1} \phi^{*} d x=c^{2} \frac{\lambda^{*}}{2}\left|\int_{0}^{1} \delta^{2} F\left(w^{*} ; \phi^{*}\right) d x\right|$, which implies that $c=$ $2 / \lambda^{*}\left|\int_{0}^{1} \delta^{2} F\left(w^{*} ; \phi^{*}\right) d x\right|>0$, since $c=0$ is rejected and provided that $\int_{0}^{1} \phi^{*} d x=1$ (we should $c>0$ otherwise $u^{*}>w^{*}$ for some $t>0$ ).

Finally $u_{2}$ needs to be estimated by the inequality

$$
u_{2}^{\prime} \leq \lambda^{*} \delta F\left(w^{*} ; u_{2}\right)+c \phi^{*}-\frac{\lambda^{*}}{2} c^{2} \delta^{2} F\left(z ; \phi^{*}\right)
$$

where $0<z=w^{*}-\xi \hat{u}<w^{*}$, for some $\xi=\xi(t) \in(0,1)$.

This implies that we need

$$
u_{2}^{\prime}(x) \leq c_{1} u_{2}(x)+c_{2} q+m_{1},
$$

where $m_{1}=c \inf _{x} \phi^{*}(x)+m_{0}=m_{0}, m_{0}=\frac{\lambda^{*}}{2} c^{2} \inf _{x} N(x),\left(\delta^{2} F\left(z ; \phi^{*}\right) \geq N(x)\right.$, $\left.0<z(x, t)<w^{*}(x)<\infty\right), c_{1}=\inf _{x}\left\{\lambda^{*} f^{\prime}\left(w^{*}\right) / I_{0}^{2}\left(w^{*}\right)\right\}, c_{2}=\sup _{x}\left\{-2 f\left(w^{*}\right) / I_{0}^{3}\left(w^{*}\right)\right\}$ and $q=\int_{0}^{1} f^{\prime}\left(w^{*}\right) u_{2} d x=I_{11}\left(w^{*}, u_{2}\right)<0$, hence

$$
u_{2} \leq \frac{c_{2} q+m_{1}}{c_{1}}\left(e^{c_{1} x}-1\right)
$$


for $u_{2}(0)=0$, which is satisfied if

$$
q=\frac{c_{2} q+m_{1}}{c_{1}} \int_{0}^{1} f^{\prime}\left(w^{*}\right)\left(e^{c_{1} x}-1\right) d x .
$$

Therefore $q=\frac{m_{1} m_{2}}{c_{1}-c_{2} m_{2}}$, for $m_{2}=\int_{0}^{1} f^{\prime}\left(w^{*}\right)\left(e^{c_{1} x}-1\right) d x$, can be estimated.

Substituting now $\psi$, which is estimated, for $\hat{u}$ in (18) (actually in the expression for $\mathrm{L}(\hat{u})$ ) and taking into account (8) and (19), we obtain (for the operator L see (18))

$$
\begin{aligned}
\mathrm{L}(\psi)= & \frac{c}{t+t_{0}}\left(\phi^{* \prime}-\lambda^{*} \delta F\left(w^{*} ; \phi^{*}\right)\right) \\
& +\frac{1}{\left(t+t_{0}\right)^{2}}\left(u_{2}^{\prime}-\lambda^{*} \delta F\left(w^{*} ; u_{2}\right)-c \phi^{*}+\frac{\lambda^{*}}{2} c^{2} \delta^{2} F\left(z ; \phi^{*}\right)\right)+O\left(\frac{1}{\left(t+t_{0}\right)^{3}}\right) \leq 0 .
\end{aligned}
$$

The last inequality holds since, on choosing $t_{0} \gg 1$, the term of order $\frac{1}{\left(t+t_{0}\right)^{2}}$ dominates the term of order $\frac{1}{\left(t+t_{0}\right)^{3}}$ (this is due to the $u^{*}$-problem and to the fact that $u^{*} \rightarrow w^{*}-$ or $(\psi+r) \rightarrow 0+$ as $t \rightarrow \infty \psi+r>0$ for all $t>0)$. Also the first bracket is zero and the second one negative, see (19).

Requiring $\psi(x, 0) \leq \hat{u}_{0}$, on choosing $t_{0} \gg 1$, finally we get that $\psi$ is a lower solution to the $\hat{u}$-problem and thus $\psi \leq \hat{u}$.

We now write $u=u^{*}+u_{1} \leq w^{*}$ and find an upper solution to the $u_{1}$-problem. The equation for $u_{1}$ is

$$
\begin{aligned}
u_{1 t} & =-u_{1 x}+\left(\lambda-\lambda^{*}\right) F\left(w^{*}\right)+\lambda\left(F(u)-F\left(w^{*}\right)\right) \\
& -\lambda^{*}\left(F\left(u^{*}\right)-F\left(w^{*}\right)\right), \quad 0<x<1, \quad t>0 .
\end{aligned}
$$

We again examine the difference $\lambda\left(F(u)-F\left(w^{*}\right)\right)$ and write $v=u-w^{*},\left(-w^{*}<v<0\right)$, $J_{1}(\epsilon)=F\left(w^{*}+\epsilon v\right), 0 \leq \epsilon \leq 1$, we have:

$$
\begin{aligned}
\lambda\left(F(u)-F\left(w^{*}\right)\right) & =\lambda\left(J_{1}(1)-J_{1}(0)\right) \\
& =\lambda\left(\frac{f^{\prime}\left(w^{*}\right) v}{I_{0}^{2}\left(w^{*}\right)}-\frac{2 f\left(w^{*}\right) I_{11}\left(w^{*} ; v\right)}{I_{0}^{3}\left(w^{*}\right)}\right)+\frac{\lambda}{2} J_{1}^{\prime \prime}\left(\xi_{1}\right) \\
& =\lambda^{*} \delta F\left(w^{*} ; v\right)+\left(\lambda-\lambda^{*}\right) \delta F\left(w^{*} ; v\right)+\frac{\lambda}{2} \delta^{2} F(z ; v) \\
& =\lambda^{*} \delta F\left(w^{*} ; v\right)+Q\left(w^{*}, z, v\right) .
\end{aligned}
$$

Also by setting $u^{*}=w^{*}-\hat{u}$ and $J_{2}(\epsilon)=F\left(w^{*}-\epsilon \hat{u}\right), 0 \leq \epsilon \leq 1$ the quantity $\lambda^{*}\left(F\left(u^{*}\right)-\right.$ $\left.F\left(w^{*}\right)\right)$ is written:

$$
\begin{aligned}
-\lambda^{*}\left(F\left(u^{*}\right)-F\left(w^{*}\right)\right) & =-\lambda^{*}\left(J_{2}(1)-J_{2}(0)\right) \\
& =\lambda^{*}\left(\frac{f^{\prime}\left(w^{*}\right) \hat{u}}{I_{0}^{2}\left(w^{*}\right)}-\frac{2 f\left(w^{*}\right) I_{11}\left(w^{*}, \hat{u}\right)}{I_{0}^{3}\left(w^{*}\right)}\right)-\frac{\lambda^{*}}{2} J_{2}^{\prime \prime}\left(\xi_{2}\right) \\
& =\lambda^{*} \delta F\left(w^{*} ; \hat{u}\right)-\frac{\lambda^{*}}{2} \delta^{2} F(\zeta ; \hat{u}),
\end{aligned}
$$

with

$J_{2}^{\prime \prime}\left(\xi_{2}\right)=\delta^{2} F(\zeta ; \hat{u})=$

$\frac{1}{I_{0}^{4}(\zeta)}\left[I_{0}^{2}(\zeta) \hat{u}^{2} f^{\prime \prime}(\zeta)-4 \hat{u} f^{\prime}(\zeta) I_{11}(\zeta, \hat{u}) I_{0}(\zeta) .-2 f(\zeta) I_{0}(\zeta) I_{22}(\zeta, \hat{u})+6 f(\zeta) I_{0}(\zeta) I_{11}^{2}(\zeta, \hat{u})\right]$

where $\zeta=w^{*}-\xi_{2} \hat{u}, \xi_{2}=\xi_{2}(t) \in(0,1)$. 
The $u_{1}$-problem (20) with relations (21), (22) now becomes

$$
\begin{gathered}
u_{1 t}=-u_{1 x}+\left(\lambda-\lambda^{*}\right) F\left(w^{*}\right)+\lambda^{*} \delta F\left(w^{*} ; v\right)+Q\left(w^{*}, z, v\right) \\
+\lambda^{*} \delta F\left(w^{*} ; \hat{u}\right)-\frac{\lambda^{*}}{2} \delta^{2} F(\zeta ; \hat{u}), \quad 0<x<1, \quad t>0, \\
u_{1}(0, t)=0, \quad t>0, \\
u_{1}(x, 0)=u_{0}(x)-u_{0}^{*}(x)=0, \quad 0<x<1,
\end{gathered}
$$

where $0<z<w^{*}, 0<\zeta<w^{*}, 0<\hat{u}<w^{*}, u<u_{1}<w^{*}$ as far as $u<w^{*}$, so that $Q\left(w^{*}, z, v\right), J_{2}^{\prime \prime}\left(\xi_{2}\right)$ are bounded from above and below. Hence, for a fixed $\lambda>\lambda^{*}$, there exists a constant $B$ such that:

$$
\begin{aligned}
u_{1 t} \leq-u_{1 x} & +\left(\lambda-\lambda^{*}\right) F\left(w^{*}\right)+\lambda^{*}\left[\frac{f^{\prime}\left(w^{*}\right) v}{I_{0}^{2}\left(w^{*}\right)}-\frac{2 f\left(w^{*}\right) I_{11}\left(w^{*}, v\right)}{I_{0}^{3}\left(w^{*}\right)}\right] \\
& +\lambda^{*}\left[\frac{f^{\prime}\left(w^{*}\right) \hat{u}}{I_{0}^{2}\left(w^{*}\right)}-\frac{2 f\left(w^{*}\right) I_{11}\left(w^{*}, \hat{u}\right)}{I_{0}^{3}\left(w^{*}\right)}\right]+\left(\lambda-\lambda^{*}\right) B .
\end{aligned}
$$

Due to the fact that $u_{1}=u-u^{*}=u-w^{*}+w^{*}-u^{*}=v+\hat{u}$, the previous relation becomes:

$$
u_{1 t} \leq-u_{1 x}+\left(\lambda-\lambda^{*}\right) F\left(w^{*}\right)+\lambda^{*}\left[\frac{f^{\prime}\left(w^{*}\right) u_{1}}{I_{0}^{2}\left(w^{*}\right)}-\frac{2 f\left(w^{*}\right) I_{11}\left(w^{*}, u_{1}\right)}{I_{0}^{3}\left(w^{*}\right)}\right]+\left(\lambda-\lambda^{*}\right) B .
$$

Now we introduce $\psi_{1}(x, t)=\left[\left(\lambda-\lambda^{*}\right) \Lambda\left(t+t_{0}\right)\right] \phi^{*}(x)$, where $\Lambda$ is a constant which is determined, so that $\psi_{1}$ can be an upper solution to the $u_{1}$-problem. Here $\phi^{*}$ again satisfies problem (8).

By substituting $-\psi_{1}$ for $u_{1}$ in the right hand side of the above relation, we get

$$
\begin{gathered}
-\psi_{1 x}+\left(\lambda-\lambda^{*}\right) F\left(w^{*}\right)+\lambda^{*}\left[\frac{f^{\prime}\left(w^{*}\right) \psi_{1}}{I_{0}^{2}\left(w^{*}\right)}-\frac{2 f\left(w^{*}\right) I_{11}\left(w^{*}, \psi_{1}\right)}{I_{0}^{3}\left(w^{*}\right)}\right]+\left(\lambda-\lambda^{*}\right) B \\
=\left(\lambda-\lambda^{*}\right) F\left(w^{*}\right)+\left(\lambda-\lambda^{*}\right) B \leq\left(\lambda-\lambda^{*}\right) \Lambda \phi^{*}=\frac{\partial \psi_{1}}{\partial t}, \\
F\left(w^{*}(x)\right)+B \leq \Lambda \phi^{*}(x), \quad \text { or } \sup _{x} F\left(w^{*}(x)\right)+B \leq \Lambda \phi^{*}(x) .
\end{gathered}
$$

Since $\sup _{x} F\left(w^{*}(x)\right)=\frac{f(0)}{f^{2}\left(w^{*}(1)\right)}$, it is enough to take

$$
\frac{f(0)}{f^{2}\left(w^{*}(1)\right)}+B=\Gamma \leq \Lambda \phi^{*}(x) \quad \text { or } \quad 0<\Gamma \leq \Lambda \inf _{x \in[\gamma, 1]} \phi^{*}(x)=\Lambda \Theta,
$$

for some $\gamma \in(0,1)$.

Choosing $\Lambda=\max \left\{\frac{w^{*}(\gamma)}{\left(\lambda-\lambda^{*}\right) t_{0} \phi^{*}(\gamma)}, \frac{\Gamma}{\Theta}\right\}$ for some $\gamma>0$ (such a $\gamma$ exists since $\phi^{*}(0)=$ 0 and $\phi^{*}(x)>0$ in $\left.(0,1]\right)$, then, $\psi_{1}$ is a "restricted" upper solution (an appropriate differential inequality in a part of the interval) for the $u_{1}$-problem in the interval $[\gamma, 1]$, [5]. Here, it must be noted that $u$ blows up globally, see relation (4b), this means that $u(x, t)$ is bounded for $(x, t) \in[0, \gamma] \times[0, T]$ for some $T<t^{*}$. In other words, $u$ is bounded in $[\gamma, 1] \times[0, T]$ (actually here we require $u<w^{*}$, see below) and hence a lower bound for $t^{*}$ can be found working in the restricted interval $[\gamma, 1]$. 
Hence, $u \leq w^{*}$ as far $\psi-\psi_{1} \geq 0$, thus we have

$$
u=u_{1}+u^{*}=w^{*}-\frac{c \phi^{*}}{t+t_{0}}-\frac{u_{2}}{\left(t+t_{0}\right)^{2}}+\left(\lambda-\lambda^{*}\right) \Lambda\left(t+t_{0}\right) \phi^{*} .
$$

The right-hand side of the above relation is no greater than $w^{*}$, as long as $\psi \geq \psi_{1}$ or

$$
\frac{c \phi^{*}}{\left(t+t_{0}\right)}+\frac{u_{2}}{\left(t+t_{0}\right)^{2}} \geq\left(\lambda-\lambda^{*}\right) \Lambda \phi^{*}\left(t+t_{0}\right), \quad x \in[\gamma, 1]
$$

Hence, $u \leq w^{*}$ as far as $\psi-\psi_{1} \geq 0$; it is enough that $u \leq w^{*}$ for any $x \in[\gamma, 1]$, for some $\gamma>0$, due to the fact that $u$ blows up globally.

Therefore, for simplicity, it is enough to choose

$$
\begin{gathered}
\frac{c \phi^{*}}{t+t_{0}} \geq\left(\lambda-\lambda^{*}\right) \Lambda \phi^{*}\left(t+t_{0}\right), \quad x \in[\gamma, 1], \\
\text { so that } \quad c \geq\left(\lambda-\lambda^{*}\right) \Lambda\left(t+t_{0}\right)^{2} .
\end{gathered}
$$

Thus we get

$$
t \leq c^{1 / 2} \Lambda^{-1 / 2}\left(\lambda-\lambda^{*}\right)^{-1 / 2}-t_{0}
$$

which for $\lambda$ sufficiently close to $\lambda^{*}\left(\lambda>\lambda^{*}\right)$, the above relation gives

$$
t \lesssim c^{1 / 2} \Lambda^{-1 / 2}\left(\lambda-\lambda^{*}\right)^{-1 / 2}=t_{l}\left(\lambda-\lambda^{*}\right)^{-1 / 2} .
$$

Hence, as long as $u=u(x, t)<w^{*}$ at $t=t_{l}\left(\lambda-\lambda^{*}\right)^{-1 / 2}$, we deduce that $t^{*}>t_{l}\left(\lambda-\lambda^{*}\right)^{-1 / 2}$ and $t_{l}\left(\lambda-\lambda^{*}\right)^{-1 / 2}$ is a lower bound for $t^{*}$ with $t_{l}=c^{1 / 2} \Lambda^{-1 / 2}$.

\section{Asymptotic and numerical estimates of $t^{*}$}

3.1. Asymptotic estimate for small $\lambda-\lambda^{*}>0$. We now examine the special case $f(s)=$ $e^{-s}$. Motivated by Section 2 we wish to find an estimate for the blow-up time $t^{*}$ to problem (1) as an asymptotic series of $\eta=\theta^{1 / 2}=\left(\lambda-\lambda^{*}\right)^{1 / 2} \ll 1, \eta>0$. We again assume that $u_{0}(x)<w^{*}(x)$ for $0<x \leq 1$, with $u_{0}(0)=w^{*}(0)=0$.

Following similar concepts to $[3,4]$, as well as motivated by numerical calculations, we consider three intervals of time, say I, II, and III. In I and III $t$ varies by $O(1)$ as $\theta=\left(\lambda-\lambda^{*}\right) \rightarrow 0$ and we expand $u \sim u^{*}+\theta v_{1}+\theta^{2} v_{2}+\ldots$ as $\theta \rightarrow 0+$, where $u^{*}$ satisfies problem (1) at $\lambda=\lambda^{*}$.

In interval II, we expand $u \sim w^{*}+\eta v_{1}+\eta^{2} v_{2}+\ldots$ as $\eta \rightarrow 0$, and making a change of time scale $t=\tau / \eta$, equation (1) gives:

$$
\eta^{2} v_{1 \tau}+\eta^{3} v_{2 \tau}+\cdots+w_{x}^{*}+\eta v_{1 x}+\eta^{2} v_{2 x}+\cdots=\lambda R(\eta) \text { as } \eta \rightarrow 0
$$

where

$$
F(u) \sim \hat{R}(x, t ; \eta):=R(\eta)=\frac{e^{-\left(w^{*}+\eta v_{1}+\eta^{2} v_{2}+\ldots\right)}}{\left(\int_{0}^{1} e^{-\left(w^{*}+\eta v_{1}+\eta^{2} v_{2}+\ldots\right)}\right)^{2} d x} \text { as } \eta \rightarrow 0 .
$$

We require an expansion for $R(\eta)$ as follows:

$$
R(\eta)=R(0)+\eta R^{\prime}(0)+\frac{\eta^{2}}{2} R^{\prime \prime}(0)+\ldots
$$

We equate the terms of zero order $\left(O(1)\right.$ or $\left.O\left(\eta^{0}\right)\right)$ and get

$$
w^{* \prime}=\lambda^{*} R(0), \quad 0<x<1, w^{*}(0)=0,
$$


where $R(0)=e^{-w^{*}} /\left(\int_{0}^{1} e^{-w^{*}} d x\right)^{2}$. Problem (25) is actually equivalent to problem (3). By looking now at the terms of $O(\eta)$ we have

$$
v_{1 x}(x, \tau)=\lambda^{*} R^{\prime}(0), \quad 0<x<1, \tau>0, \quad v_{1}(0, \tau)=0, \quad \tau>0,
$$

where $R^{\prime}(0)=\delta F\left(w^{*} ; v_{1}\right)=-\frac{e^{-w^{*}} v_{1}}{\left(\int_{0}^{1} e^{-w^{*}} d x\right)^{2}}+\frac{2 e^{-w^{*}} \int_{0}^{1} e^{-w^{*}} v_{1} d x}{\left(\int_{0}^{1} e^{-w^{*}} d x\right)^{3}}$.

Problem (26) has the form of problem (8), thus we can write

$$
v_{1}(x, \tau)=a(\tau) \phi^{*}(x),
$$

where now we normalize $\phi^{*}$ according to $\int_{0}^{1} \phi^{* 2}(x) d x=1$ and we denote the normalized $\phi^{*}$ again by $\phi^{*}$. Looking next at the $O\left(\eta^{2}\right)$ terms we have

$$
v_{1 \tau}+v_{2 x}=R(0)+\frac{\lambda^{*}}{2} R^{\prime \prime}(0),
$$

which becomes

$$
\begin{aligned}
v_{1 \tau}+v_{2 x} & =\frac{e^{-w^{*}}}{S_{0}^{2}\left(\phi^{*}\right)}+\frac{2 \lambda^{*} e^{-w^{*}} v_{1} S_{1}\left(v_{2}\right)}{S_{0}^{3}\left(\phi^{*}\right)}+\frac{2 \lambda^{*} e^{-w^{*}} S_{1}\left(v_{2}\right)}{S_{0}^{3}\left(\phi^{*}\right)} \\
& -\frac{\lambda^{*} e^{-w^{*}} S_{2}\left(v_{2}\right)}{S_{0}^{3}\left(\phi^{*}\right)}+\frac{3 \lambda^{*} e^{-w^{*}} S_{1}^{2}\left(v_{2}\right)}{S_{0}^{4}\left(\phi^{*}\right)}
\end{aligned}
$$

where now we denote $I_{\nu k}\left(w^{*}, v\right)=(-1)^{\nu} S_{k}(v)$ with $S_{k}(v)=\int_{0}^{1} e^{-w^{*}} v^{k} d x, k=0,1,2,3$, and $S_{k}\left(\phi^{*}\right)=S_{k}$.

Multiplying (28) by $\phi^{*}$, integrating over $[0,1]$, using (27) and normalizing $\phi^{*}$, we obtain

$$
\begin{array}{r}
\dot{a}(\tau)=\int_{0}^{1}\left(-\phi^{* \prime}-\frac{\lambda^{*} e^{-w^{*}} \phi^{*}}{S_{0}^{2}}+\frac{2 \lambda^{*} e^{-w^{*}} S_{1}}{S_{0}^{3}}\right) v_{2} d x+\frac{S_{1}}{S_{0}^{2}}+ \\
\lambda^{*} \frac{a^{2}(\tau)}{2 S_{0}^{4}}\left(S_{3} S_{0}^{2}-6 S_{1} S_{2} S_{0}+6 S_{1}^{3}\right) .
\end{array}
$$

Since the quantity inside the integral is zero (the linearized problem), and setting $S=S_{3} S_{0}^{2}-6 S_{1} S_{2} S_{0}+6 S_{1}^{3}$, equation (29) can be written as

$$
\dot{a}(\tau)=\frac{S_{1}}{S_{0}^{2}}+\lambda^{*} \frac{S}{2 S_{0}^{4}} a^{2}(\tau), \quad \tau>0, a(\tau) \rightarrow-\infty \text { as } \tau \rightarrow 0,
$$

(this choice of the initial condition as $\tau \rightarrow 0+$ gives constant of integration $-\pi / 2$ ).

Returning to the time variable $t$, problem (30) becomes

$$
A(t)=\left(\frac{B}{K}\right)^{-1 / 2} \tan \left[t\left(\lambda-\lambda^{*}\right)^{1 / 2}(B K)^{1 / 2}-\frac{\pi}{2}\right],
$$

where $K=\lambda^{*} S / 2 S_{0}^{4}, B=S_{1} / S_{0}^{2}$.

Because $u=w^{*}+\eta v_{1}+\ldots$ and $v_{1}(x, t)=A(t) \phi^{*}(x)$, it is obvious that $u$ ceases to exist at time

$$
t^{*} \sim t_{b}=t_{u}\left(\lambda-\lambda^{*}\right)^{-\frac{1}{2}}
$$

where $t_{u}=\pi(4 B K)^{-1 / 2}$ and $t_{b}$ is the blow-up time of $a(\tau)=a\left(t\left(\lambda-\lambda^{*}\right)^{1 / 2}\right)=A(t)$. 
3.2. Numerical estimates. We solve problem (1) by using a two-step up-wind scheme. For the linear terms we apply the usual form of the scheme:

$$
v_{j}^{n+1}=u_{j}^{n}-r\left(u_{j}^{n}-u_{j-1}^{n}\right)+\lambda F\left(u_{j}^{n}\right),
$$

where $u_{j}^{n}$ is the temperature at the $n$th time level and at the $j$ th space grid, $r=\delta t / \delta x$ and the non-local term $F\left(u_{j}^{n}\right)$ is evaluated at the $n$th time step. For this term we have

$$
F\left(u_{j}^{n}\right)=\frac{f\left(u_{j}^{n}\right)}{\left(\int_{0}^{1} f\left(u_{j}^{n}\right) d x\right)^{2}} .
$$

The integral in the denominator is evaluated by Simpson's rule. In the next step we evaluate $w$

$$
w_{j}^{n+1}=u_{j}^{n}-r\left(v_{j}^{n+1}-v_{j-1}^{n+1}\right)+\lambda F\left(v_{j}^{n+1}\right) .
$$

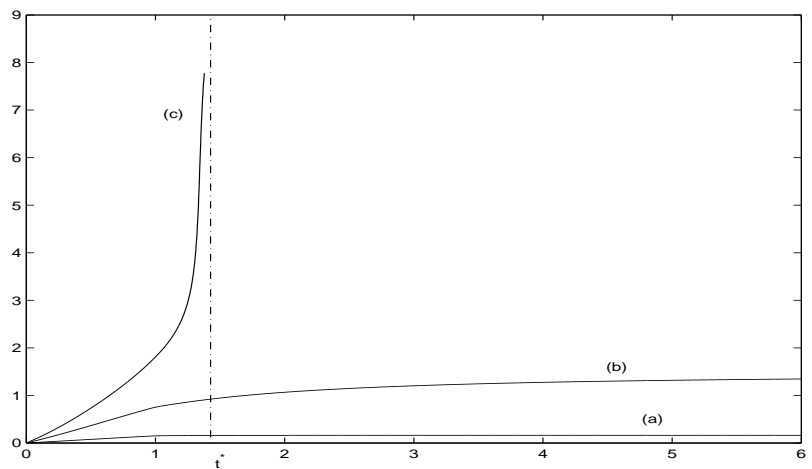

Fig. 2. Numerical solution to problem (1). We plot $\max _{x}(u(x, t))=u(1, t)=M(t)$, for $x$ in $[0,1]$ against time for $\delta x=0.033, \delta t=0.002$ (the upper curve, (c), corresponds to $\lambda=1.1476>\lambda^{*}=$ 0.6476 , the intermediate, (b), to $\lambda=\lambda^{*}$ and the lower one, (a), to $\lambda=0.1476<\lambda^{*}$ ). Also the dash-dotted axis corresponds to the asymptotic estimate of the blow-up time $t^{*} \sim 1.3367$ for $\lambda=1.1476$. To obtain this estimate we numerically calculate $w^{*}$ by an iteration scheme and then we solve the equation for $\phi^{*}$ using the appropriate normalization.

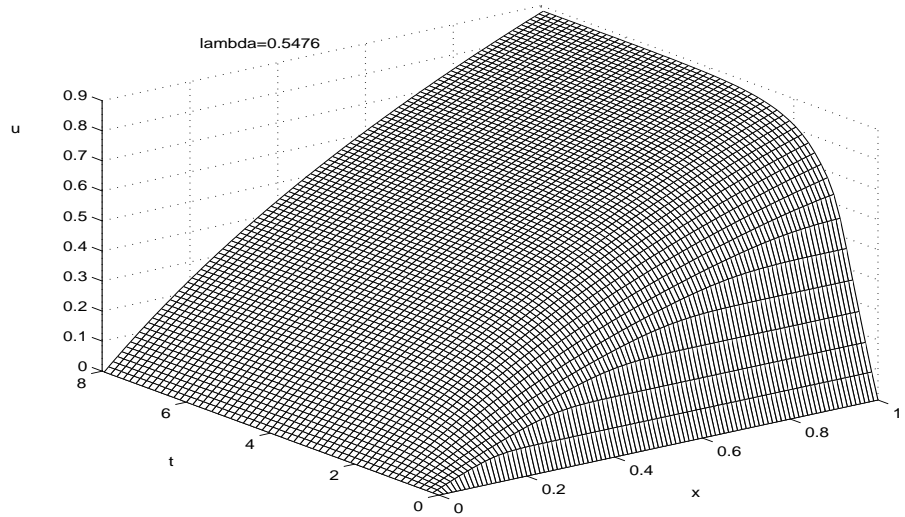

Fig. 3. Numerical solution to problem (1) for $\lambda=0.5476<\lambda^{*}$. 
Finally, $u$ at the $(n+1)$ th time step is approximated by

$$
u_{j}^{n+1}=\frac{1}{2}\left(v_{j}^{n+1}+w_{j}^{n+1}\right) .
$$

In Figure 2 we use this scheme to solve the problem numerically for $f(u)=e^{-u}$ and taking $u(x, 0)=0$. We see that for $\lambda<\lambda^{*}$ the solution $u$ tends to a steady state, for $\lambda=\lambda^{*}$ the behaviour is similar, and for $\lambda>\lambda^{*}$ the solution blows up (the decay is faster for $\lambda<\lambda^{*}$ than it is for $\lambda=\lambda^{*}$ ). More precisely, in Figure 2 the maximum of solutions is plotted against time.

In Figure 3, we plot the numerical solution of $u$ for $\lambda=0.5476$.

4. Discussion. In the present work, we estimate the blow-up time $t^{*}$ of the solution to problem (1). In this mathematical model, the blow-up time represents the time when the food is burnt. Similar estimates are also known for local (the reaction diffusion problem) as well as for non-local (the Ohmic heating problem) problems $[3,4]$. Here the results are obtained for the case where there exists a steady-state solution $w^{*}=w\left(x ; \lambda^{*}\right)$ at $\lambda=\lambda^{*}$, for $0<\lambda-\lambda^{*} \ll 1$ and nonnegative initial data. The methods applied are comparison and asymptotic techniques, as well as numerical computations.

Our main estimates, for given $\lambda, \lambda^{*}$ and $0<\lambda-\lambda^{*} \ll 1$, are: upper bound $\epsilon+$ $c_{1} \ln \left[c_{2}\left(\lambda-\lambda^{*}\right)^{-1}\right]$; lower bound $c_{3}\left(\lambda-\lambda^{*}\right)^{-1 / 2}$; asymptotic estimate $t^{*} \sim c_{4}\left(\lambda-\lambda^{*}\right)^{-\frac{1}{2}}$ as $\lambda \rightarrow \lambda^{*}+$; some numerical results are also presented.

A more substantial work will appear elsewhere, where in addition the case of $0<\lambda<$ $\lambda^{*}$ and initial data greater than the greatest steady-state solution will be studied.

\section{References}

[1] J. A. Carrillo, On a non-local elliptic equation with decreasing nonlinearity arising in plasma physics and heat conduction. Nonlinear Analysis TMA 32 (1998), 97-115.

[2] P. Freitas and M. Grinfeld, Stationary solutions of an equation modelling Ohmic heating, Appl. Math. Lett. 7, (1994), No. 3, 1-6.

[3] N. I. Kavallaris, C. V. Nikolopoulos and D. E. Tzanetis, Estimates of blow-up time for a non-local problem modelling an Ohmic heating process, Euro. J. Appl. Math. 13 (2002), $337-351$.

[4] A. A. Lacey, Mathematical analysis of thermal runaway for spatially inhomogeneous reactions, SIAM J. Appl. Math. 43 (1983), 1350-1366.

[5] A. A. Lacey and D. Tzanetis, Global existence and convergence to a singular steady state for a semilinear heat equation, Proc. Royal Soc. Edinburgh Sect. A 105 (1986), 289-305.

[6] A. A. Lacey, Thermal runaway in a non-local problem modelling Ohmic heating. Part I: Model derivation and some special cases, Euro. J. Appl. Math. 6 (1995), 127-144.

[7] A. A. Lacey, Thermal runaway in a non-local problem modelling Ohmic heating. Part II: General proof of blow-up and asymptotics of runaway, Euro. J. Appl. Math. 6 (1995), 201-224.

[8] A. A. Lacey, D. E. Tzanetis and P. M. Vlamos, Behaviour of a nonlocal reactive convective problem modelling Ohmic heating of foods, Quart. J. Mech. Appl. Math. 5 (1999), 623-644.

[9] D. H. Sattinger, Monotone methods in nonlinear elliptic and parabolic boundary value problems, Indiana Univ. Math. J. 21 (1972), 979-1000. 
[10] D. E. Tzanetis, Blow-up of radially symmetric solutions of a non-local problem modelling Ohmic heating, Elec. J. Diff. Eqns. 2002, No. 11, 1-26. 